\title{
Three-Layered Optic Disc Hemorrhages in Idiopathic Intracranial Hypertension
}

\author{
Irina Sverdlichenko ${ }^{a}$ Jonathan A. Micieli $b, c, d$ \\ ${ }^{\mathrm{a}}$ Faculty of Medicine, University of Toronto, Toronto, ON, Canada; ${ }^{\mathrm{b}}$ Department of \\ Ophthalmology \& Vision Sciences, University of Toronto, Toronto, ON, Canada; 'Division \\ of Neurology, Department of Medicine, University of Toronto, Toronto, ON, Canada; \\ ${ }^{\mathrm{d} K e n s i n g t o n}$ Vision and Research Centre, Toronto, ON, Canada
}

\begin{abstract}
Keywords
Idiopathic intracranial hypertension $\cdot$ Optic disc hemorrhage $\cdot$ Retinal hemorrhage $\cdot$ Multilayered
\end{abstract}

\begin{abstract}
Papilledema related to idiopathic intracranial hypertension (IIH) may present with optic disc hemorrhaging. The significance and characteristics of these hemorrhages still remains to be clarified. Here, we present 2 unique cases with severe unilateral optic disc hemorrhaging in all 3 retinal layers (preretinal, intraretinal, and subretinal) and a good visual outcome. A 42- and a 44-year-old woman presented with signs and symptoms of raised intracranial pressure. Visual acuity was normal and Humphrey visual field testing showed only mild vision loss with enlarged blind spots. Dilated fundus examination revealed moderate papilledema in both eyes with severe optic disc hemorrhaging in the left eye in the first patient and the right eye in the second patient. The optic disc hemorrhaging occurred in all 3 retinal layers (preretinal, intraretinal, and subretinal), and the fellow eyes had only minimal hemorrhage. All patients had normal magnetic resonance imaging/magnetic resonance venography, and lumbar punctures revealed elevated opening pressures and normal cerebrospinal fluid contents. The papilledema resolved after 4 months in both cases with medical therapy. Optic disc hemorrhaging may be severe in patients with $\mathrm{IIH}$, but this does not necessarily confer a poor visual outcome. The differential diagnosis of hemorrhages in all 3 retinal layers may be expanded to include $\mathrm{IH}$.
\end{abstract}

\section{Karger!}




\section{Introduction}

Idiopathic intracranial hypertension (IIH) is a condition of raised intracranial pressure (ICP) primarily seen in young, obese women and does not have an identifiable cause. Optic disc hemorrhages (ODH) have been reported in about one-third of patients with this condition [1] but may not have any prognostic value when controlling for the severity of papilledema [2]. We report 2 unique cases of unilateral severe hemorrhaging in all 3 retinal layers in $\mathrm{IIH}$ patients with good visual outcomes.

\section{Patient 1}

A 42-year-old obese woman presented to her optometrist after she noticed "after images" following bright light exposure in her left eye (OS). She also noticed transient blurred vision with position changes but denied any regular headaches or pulsatile tinnitus. She denied ever having a severe onset headache. She was referred to neuro-ophthalmology and was found to have a visual acuity of 20/20 in both eyes. Humphrey 24-2 SITA-Fast visual fields showed nonspecific depressed points right eye (OD; MD -6.18 dB) and showed an enlarged blind spot OS (MD -4.84 dB). Dilated fundus examination showed moderate optic disc edema in both eyes with preretinal, intraretinal, and subretinal hemorrhages around the left optic disc (shown in Fig. 1a). Her blood pressure measured 140/100 and her hemoglobin was normal. Magnetic resonance imaging/magnetic resonance venography was performed and showed signs of raised ICP without a secondary cause. Lumbar puncture showed an opening pressure of $32 \mathrm{~cm}$ of water and normal cerebrospinal fluid (CSF) contents. She was started on acetazolamide $500 \mathrm{mg}$ twice-daily (BID) and telmisartan $40 \mathrm{mg}$ daily and the optic disc edema improved and resolved after 4 months with significant weight loss. She maintained normal visual function and the ODH resolved at 3 months. The final Humphrey mean deviation was $-3.42 \mathrm{~dB}$ OD and $-2.98 \mathrm{~dB}$ OS.

\section{Patient 2}

A 44-year-old obese woman with a medical history of iron-deficiency anemia (pretreatment hemoglobin of $8.8 \mathrm{~g} / \mathrm{dL}$ ) presented with blurred vision in her right eye and new headaches over 1 month. She described the headaches as holocephalic, rated them 4-5 out of 10 (where 1 is minimal intensity and 10 is maximal intensity) without any diurnal or positional variation. She had associated intermittent nausea, but no vomiting. She denied any characteristics consistent with a thunderclap headache. She initially saw her optometrist and was referred to neuroophthalmology for bilateral optic disc edema. Examination revealed a visual acuity of 20/30 OD and 20/20 OS, and Humphrey visual field testing showed an enlarged blind spot OD (MD -4.60 $\mathrm{dB}$ ) and was normal OS (MD -2.10 dB). Slit-lamp examination revealed a posterior subcapsular cataract in the OD and dilated fundus examination showed moderate optic disc edema in both eyes with hemorrhages in all 3 retinal layers in the OD (shown in Fig. 1b). Her blood pressure was $128 / 78$, and her hemoglobin was found to be $9.0 \mathrm{~g} / \mathrm{dL}$ with mean corpuscular volume of $70.4 \mathrm{fL}$. Magnetic resonance imaging/magnetic resonance venography of the head was normal apart from signs of raised ICP, and lumbar puncture showed an opening pressure of $34 \mathrm{~cm}$ of water with normal CSF contents. She was started on acetazolamide $500 \mathrm{mg}$ BID and continued her recent supplementation with ferrous fumarate. With significant weight loss, her optic disc edema resolved after 4 months, and she continued to maintain normal visual function. The final Humphrey mean deviation was $-2.80 \mathrm{~dB}$ OD and $-0.72 \mathrm{~dB}$ OS.

\section{Karger'}



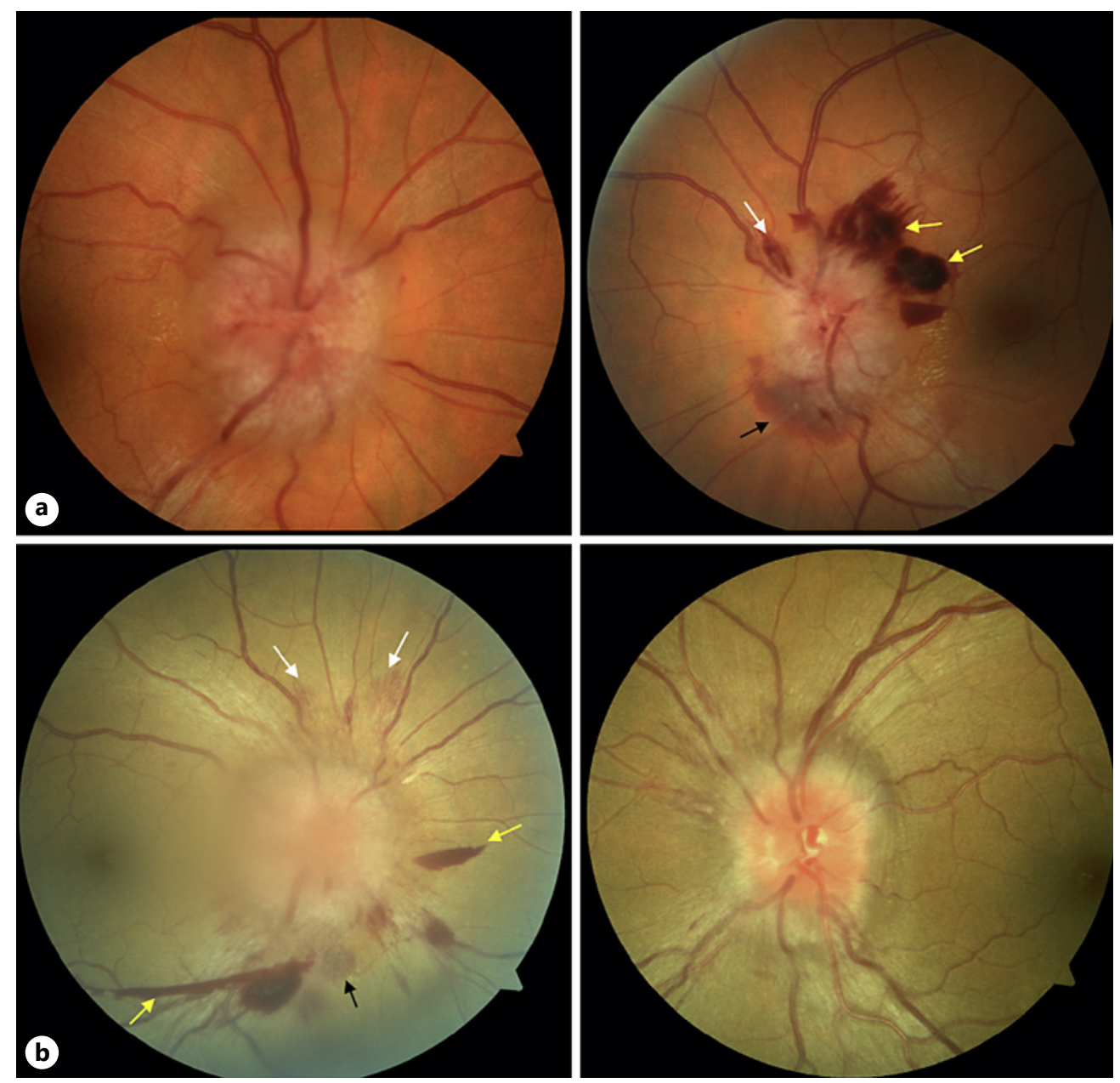

Fig. 1. Fundus photographs for patients 1 (a) and 2 (b). Both patients show bilateral moderate optic disc edema. ODH in all 3 retinal layers in the OS of patient 1 and OD of patient 2. Preretinal (yellow arrow), intraretinal (white arrow), and subretinal (black arrow) hemorrhages are indicated. ODH, optic disc hemorrhages; OS, left eye; OD, right eye.

\section{Conclusion}

We report a unique observation of 2 patients with IIH and mild vision loss at presentation with severe ODH in all 3 retinal layers in only one eye. The IIH Treatment Trial assessed hemorrhages in prospectively recruited IIH patients with mild vision loss and found that $31.6 \%$ of subjects had $\mathrm{ODH}$, and these were most commonly in the nerve fiber layer $(27.2 \%)$ and rarely subretinal (4.4\%) [1]. No individual in the study had a preretinal hemorrhage. They reported that $\mathrm{ODH}$ correlated with the severity of papilledema and was more frequently found in treatment failure subjects, suggesting that they had prognostic value. However, the predictive value of ODH was not evaluated independently of papilledema severity. A larger study of 360 consecutive IIH patients of all severities found that ODH was associated with the severity of papilledema but did not correlate with final visual outcome when controlling for papilledema severity [2]. Our 2 cases support the notion that $\mathrm{ODH}$ do not have predictive value as both patients had severe hemorrhaging at presentation, but an excellent visual outcome.

Multilayered $\mathrm{ODH}$ in $\mathrm{IIH}$ are rare and were not found in patients enrolled in the IIH Treatment Trial. Subretinal hemorrhages [3] and preretinal hemorrhages [4] have previously 
been identified in IIH patients in isolation, and we identified one case with a combination of preretinal and intraretinal hemorrhages in one eye [5]. The eye that had multilayered hemorrhages had only mild vision loss at presentation with a mean deviation of $-4.00 \mathrm{~dB}$ and an enlarged blind spot on Humphrey visual field testing and had a good visual outcome. Because most of the vessels on the optic disc are venous in nature, their thin walls are prone to rupture as optic nerve fibers swell and result in venous stasis. As the papilledema increases in severity, the central retinal vein may be obstructed, increasing the likelihood of more severe hemorrhaging [5]. The acuity of the papilledema may also be an important factor in producing hemorrhaging in addition to the severity of papilledema. The predominantly venous nature of the bleeding may explain why the hemorrhages are not independent predictors of visual outcome, unlike glaucoma where the ODHs are thought to be arterial in nature and related to dysfunctional autoregulation of blood flow to the optic nerve [6].

Both patients in the current report had unilateral severe optic disc hemorrhaging. We hypothesize that this was related to asymmetric transmission of CSF pressure to the optic nerve head. An acute rise in ICP may have also occurred in those eyes. It is known that papilledema can be highly asymmetric or even unilateral in IIH. Bidot et al. [7] reported that $3.6 \%$ of consecutive IIH patients had very asymmetric papilledema (defined as a difference of 2 or more Frisen grades between eyes) at initial evaluation. They suspected that this was related to differences in the size of the bony optic canals. In a larger study, the same group found that poor visual function and severe papilledema or optic atrophy were associated with a larger optic canal [8]. Contrarily, Farrokhi et al. [9] found asymmetric papilledema using the same definition in a larger proportion of patients $(18.6 \%$ of 59 IIH patients) but did not find bony optic canal size was different in the patients with asymmetric papilledema. Regardless of the exact mechanism, asymmetry in papilledema is likely a result of compartmentation of the perioptic subarachnoid spaces resulting in a "bottleneck" of CSF flow between the perioptic subarachnoid spaces and the suprasellar cistern [10].

Outside of the optic nerve, there is a differential diagnosis of retinal hemorrhages in all 3 retinal layers. This includes retinal arterial microaneurysms, age-related macular degeneration (AMD), and retinal angiomatous proliferation. The mechanism of hemorrhage in retinal arterial microaneurysms involves atrophy of the muscularis layer of the arterial wall, thinning, and fibrosis causing reduced elasticity [11]. This increases susceptibility to arterial dilation from raised hydrostatic pressure such as hypertension. Retinal angiomatous proliferation is considered a variant of neovascular AMD with intraretinal capillary proliferation. The origin of the neovascularization in both instances is the choroid, but multilayered hemorrhages may develop during various stages of vasogenesis [12]. Although rare, our cases suggest that IIH should be added to the differential diagnosis of three-layered ODH. Our patients also had coexisting anemia and hypertension, which may have played a role in the development of the $\mathrm{ODH}$. Although retinal hemorrhages have been reported in $\mathrm{IIH}$, we could not find any previous studies demonstrating hemorrhages in multiple layers or the prognostic value of these types of hemorrhages in IIH [13].

In conclusion, IIH may result in severe $\mathrm{ODH}$ involving all 3 retinal layers even in patients with mild visual dysfunction at presentation. Our cases support previous literature in that the hemorrhaging does not have prognostic value as both our patients had an excellent visual outcome.

\section{Acknowledgement}

This manuscript does not include any nonauthor contributors to acknowledge.

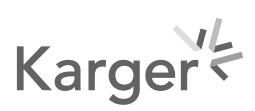


Sverdlichenko and Micieli: Three-Layered Retinal Hemorrhages in IIH

\section{Statement of Ethics}

The patients gave oral and written consent to publish the data and images in this report. The report does not include any personal information that could identify the patients directly or indirectly. All medical interventions have been carried out according to the latest protocols of therapy. The study complies with the guidelines for human studies and was conducted ethically in accordance with the World Health Organization Declaration of Helsinki. This study protocol was reviewed and the need for approval was waived by the University of Toronto Research Ethics Board. This exemption was on the basis that the information in the case report was not obtained through a systematic investigation and was not collected with a prior research intent. Furthermore, the information presented in the case report is not considered to be generalizable.

\section{Conflict of Interest Statement}

The authors have no conflicts of interest to declare.

\section{Funding Sources}

This manuscript did not receive any funding.

\section{Author Contributions}

I.S. acquired the data, drafted the original manuscript, and gave final version approval. J.M. developed the original idea for the manuscript, provided critical revisions for the manuscript, and gave final approval for the version to be published.

\section{Availability of Data and Material}

All data generated during this study are included in this article. Further enquiries can be directed to the corresponding author.

\section{References}

1 Wall M, Thurtell MJ. Optic disc haemorrhages at baseline as a risk factor for poor outcome in the Idiopathic Intracranial Hypertension Treatment Trial. Br J Ophthalmol. 2017 Sep;101(9):1256-60.

2 Micieli JA, Bruce BB, Vasseneix C, Blanch RJ, Berezovsky DE, Peragallo JH, et al. Optic nerve appearance as a predictor of visual outcome in patients with idiopathic intracranial hypertension. Br J Ophthalmol. 2019 Oct; 103(10):1429-35.

3 McCasland BJ, Mendicino ME, Newman NJ. Subretinal haemorrhage in idiopathic intracranial hypertension. $\mathrm{Br}$ J Ophthalmol. 1999;83(7):883-4.

4 Biousse V, Rucker JC, Vignal C, Crassard I, Katz BJ, Newman NJ. Anemia and papilledema. Am J Ophthalmol. 2003 Apr;135(4):437-46.

5 Raevis J, Elmalem VI. Pseudotumor cerebri syndrome causing a terson like syndrome. Am J Ophthalmol Case Rep. 2020 Dec;20:100993.

6 Drance S, Anderson DR, Schulzer M. Risk factors for progression of visual field abnormalities in normaltension glaucoma. Am J Ophthalmol. 2001 Jun;131(6):699-708.

7 Bidot S, Bruce BB, Saindane AM, Newman NJ, Biousse V. Asymmetric papilledema in idiopathic intracranial hypertension. J Neuroophthalmol. 2015 Mar;35(1):31-6. 
Sverdlichenko and Micieli: Three-Layered Retinal Hemorrhages in IIH

8 Bidot S, Clough L, Saindane AM, Newman NJ, Biousse V, Bruce BB. The optic canal size is associated with the severity of papilledema and poor visual function in idiopathic intracranial hypertension. J Neuroophthalmol. 2016 Jun;36(2):120-5.

9 Farrokhi Y, Sharif Kashani S, Aghsaei Fard M, Pakdel F, Yadegari S. Optic canal size in idiopathic intracranial hypertension and asymmetric papilledema. Clin Neurol Neurosurg. 2019 Sep;184:105376.

10 Killer HE, Subramanian PS. Compartmentalized cerebrospinal fluid. Int Ophthalmol Clin. 2014 Winter;54(1): 95-102.

11 Townsend-Pico WA, Meyers SM, Lewis H. Indocyanine green angiography in the diagnosis of retinal arterial macroaneurysms associated with submacular and preretinal hemorrhages: a case series. Am J Ophthalmol. 2000 Jan;129(1):33-7.

12 Gross NE, Aizman A, Brucker A, Klancnik JM Jr, Yannuzzi LA. Nature and risk of neovascularization in the fellow eye of patients with unilateral retinal angiomatous proliferation. Retina. 2005 Sep;25(6):713-8.

13 Nichani P, Micieli JA. Retinal manifestations of idiopathic intracranial hypertension. Ophthalmol Retina. 2020 Aug 26;5(5):429-37. 